\title{
Viajeros a África
}

\author{
Clínica Las Condes, \\ Santiago, Chile. \\ Centro del Viajero. \\ Recibido: 10 de enero de 2011.
}

Correspondencia a: Guillermo Acuña L. gacuna@netline.cl
Guillermo Acuña L.

\author{
Travellers to Africa
}

omo es muy fácil de entender, cada región geográfica tiene sus propias peculiaridades y cuando uno habla de un continente tan extenso como África, es muy importante entender la diversidad de climas, geografía, realidades políticas y económicas de cada país y no aplicar un solo estándar para un viajero que concurre diciendo "voy a África....". Como en cada consulta de Medicina del Viajero, el itinerario, la actividad a desarrollar y el tiempo de permanencia son factores muy importantes de analizar junto con las condiciones especiales del viajero (edad, sexo, condiciones especiales tales como el embarazo, o patologías concomitantes).

En forma arbitraria y con el objeto de diferenciar grandes regiones del continente, dividiremos la nota en viajeros al Norte de África (región Mediterránea y desierto Sahara), región Sub-Sahariana, África Ecuatorial y Cono sur de África.

\section{África del Norte}

Consideramos en esta región a: Marruecos, Argelia; Túnez, Libia, Egipto y el territorio de Sahara del Oeste.

En esta región, como en muchas otras del mundo, la principal precaución es la de diarrea del viajero, es decir, el cuidado de la bebida y comida. Para viajeros que no hayan tenido hepatitis $\mathrm{A}$ en su niñez o no hayan sido vacunados, se sugiere vacunar, en especial si la estadía es prolongada. Así mismo, debe ofrecerse la vacuna contra fiebre tifoidea.

En estadías prolongadas, en especial con niños pequeños, es oportuno ofrecer un esquema de vacuna anti-rábica pre-exposición, al igual que viajeros de aventura que recorrerán cuevas- entraña el riesgo de murciélagos infectados- o harán extensas caminatas. Todas las mordeduras o heridas provocadas por perros en esta región deben ser consideradas de riesgo y con indicación de vacunación post-exposición.

Debe actualizarse la vacunación de tétanos/difteria.

No hay malaria en la región ni tampoco fiebre amarilla. Sin embargo, Argelia, Túnez, Libia y Egipto exigen carné de vacunación si el viajero proviene (originalmente o en tránsito) de un país endémico de fiebre amarilla. Una lista de estos países se puede encontrar en el siguiente link: http://wwwnc.cdc.gov/travel/yellowbook/2010/ chapter-2/yellow-fever-vaccine-requirements-andrecommendations.aspx Como se aprecia, incluye a todos los países de Sudamérica, excepto Uruguay y Chile. Egipto tiene un listado un poco diferente al de la OMS en el que incluye a Belice, Costa Rica y Zambia y omite a Argentina, Paraguay y Mauritania.

El principal problema para el visitante en esta región son las infecciones digestivas. Debe recalcarse las precauciones de la bebida y comida. Se sugiere dar indicaciones de auto-tratamiento como dieta, reposo, inhibidores de la motilidad intestinal y antimicrobianos, explicando cuándo y cómo ingerirlos.

\section{África Sub-Sahariana}

Incluye: Mauritania, Mali, Niger, Chad, Sudán y Etiopía.

\section{Fiebre amarilla}

Existe riesgo de fiebre amarilla, la vacuna es exigida para entrar a Mali y Níger, y se pide si se viene de países endémicos, en Mauritania, Chad, Sudan y Etiopía. En todo caso, es recomendada a todo viajero a la zona que va a salir de zonas urbanas.

\section{Hepatitis $A$}

La vacuna está recomendada a todos los viajeros no inmunes a: Chad, Etiopía, Mauritania, Niger y Sudán.

\section{Fiebre tifoidea}

Vacunación recomendada a los viajeros a Chad, Etiopía, Mauritania, Niger y Sudán.

\section{Hepatitis B}

Vacunación recomendada a viajeros con estadías prolongadas, viajeros de aventura, posibilidad de tatuajes, trabajos dentales o acupuntura o viajeros que con alta probabilidad requieran de atención médica en algún centro de estos países.

\section{Rabia}

Se debe presumir riesgo en la mayor parte de los siguientes países: Chad, Etiopía, Mali, Mauritania, Níger 
y Sudán. Se recomienda la vacunación pre-exposición, en especial a niños pequeños. También para viajeros de aventura, mochileros y exploradores de cuevas. Todas las mordeduras y erosiones producidas por perros, chacales o mangostas deben considerarse seriamente y colocar el total de dosis de vacunas post-exposición.

\section{Meningitis (enfermedad) meningocóccica}

Etiopía, Sudán. Se recomienda vacuna cuadrivalente (A; C; Y; W-135) a todos los viajeros, durante todo el año, en especial si se tendrá contacto prolongado con la población local y para todos los niños y trabajadores de la salud. Se ha reportado actividad en la mayor parte de estos países.

Chad, Mali, Mauritania, Níger. Se recomienda vacuna cuadrivalente (A; C; Y; W-135), a viajeros durante el periodo seco, de diciembre a junio. Se ha reportado actividad, especialmente en la parte sur de estos países.

\section{Vacunas de rutina}

\section{Tétanos/difteria/ pertussis}

Los adultos que no hayan recibido una dosis previa de vacuna pertussis acelular (hoy en día, en la práctica esta situación es universal) deben recibir por una vez la vacuna Tdap en vez del refuerzo con $\mathrm{Td}$.

\section{Sarampión}

Vacuna indicada en aquellos nacidos después de 1965, sin historia de la enfermedad o que no hayan recibido dos dosis de vacuna.

\section{Poliomielitis}

Debe cumplirse con vacunación durante la infancia y una dosis de refuerzo en el adulto (inactivada, parenteral).

\section{Malaria}

Chad, Mali, Niger y Sudán: Existe predominantemente Plasmidium falciparum, en todo el país, incluyendo áreas urbanas. Deben tomarse medidas para evitar picadura de insectos, en especial en las horas del atardecer y nocturna. Quimioprofilaxis: se efectuará con mefloquina (Lariam $\left.{ }^{\circledR}\right)$, doxiciclina o atavacuona/proguanil (Malarone $\left.{ }^{\circledR}\right)$. Debe considerarse llevar dosis de antimaláricos para autotratamiento.

Etiopía: Predomina P. falciparum en todo el país incluyendo áreas urbanas, excepto Addis Abeba y altitudes sobre los $2.500 \mathrm{mts}$.

Mauritania: Existe predominantemente P. falciparum, en todo el país, excepto en las provincias de DakheletNouadhibou, Adrar y Tris Zemmour

\section{Tuberculosis}

En esta área, los países tienen una incidencia de más de 100 casos por 100 mil habitantes. Los viajeros que van a estar más de un mes en alguno de estos países deben tener un PPD antes del viaje para chequearlo en su retorno.

\section{Otras enfermedades}

Esquistosiomasis, oncocerquiasis, Ébola y Fiebre de Lassa son enfermedades presentes en el área. Rara vez son problema para el viajero, pero debe advertirse que se evite el baño en aguas dulces y tener precaución por las picaduras de insectos.

\section{África Ecuatorial}

Incluye: Senegal, Gambia, Guinea-Bissau, Guinea, Costa de Marfil, Ghana, Togo, Benin, Burkina Faso, Nigeria, Camerún, República Central Africana, Uganda, Kenya, Gabón, Congo, República Democrática del Congo, Burundi, Rwanda, Tanzania, Angola, Zambia, Malawi, Mozambique.

\section{Posibles riesgos}

Prevenibles por vacunas: Cólera, hepatitis A y B, meningitis (enfermedad) meningocóccica, poliomielitis, rabia, influenza, fiebre tifoidea, fiebre amarilla.

Otras enfermedades (no prevenibles por vacunas): Malaria, tripanosomiasis africana, influenza aviar, dengue, leishmaniasis, loaiasis, oncocerquiasis, fiebre del Rift Valley, esquistosomiasis, diarrea del viajero, tuberculosis, fiebres virales hemorrágicas.

Todo este grupo de países tienen riesgo de fiebre amarilla, todos los viajeros deben tener su inmunización al día. Algunos de estos países la solicitan como obligatoria para poder ingresar. También es recomendable la vacunación contra hepatitis A para todo viajer o que no haya tenido la enfermedad clínica o tenga inmunización incompleta. La vacunación contra hepatitis $\mathrm{B}$ se recomienda a todos aquellos que tendrán una estadía prolongada o que tengan alta probabilidad de consultar algún centro de salud, como actividades de turismo aventura con posibilidades de trauma o condiciones mórbidas del viajero que lo haga proclive a consultas médicas pudiendo necesitar inyecciones $\mathrm{u}$ otros.

Se recomienda inmunización pre-exposición de rabia para estadías prolongadas, con prioridad a niños. También en viajeros de aventura, mochileros, exploradores de cavernas.

\footnotetext{
Meningitis (enfermedad) meningocóccica: Vacuna cuadrivalente ( $A, C, Y, W$-135)

Indicada en viajes a: Benin, Costa de Marfil, Burkinia Faso, República Central Africana, Gambia, Ghana, Guinea, Guinea-Bissau, Nigeria, Senegal, Togo, Benin, Costa de Marfil, República Democrática del Congo.
} 
Se recomienda a viajeros en la estación seca (diciembre-junio), especialmente si se va a tener contacto importante con población local, $\mathrm{y}$ a todos los niños y trabajadores del área de la salud (todo el año).

Camerún: recomendada especialmente para visitantes al área norte del país.

Uganda: se recomienda a visitantes del área norte (fronteras con Sudán y Kenya). Para el resto de país se recomienda para aquellos que tendrán amplio contacto con la población local y trabajadores del área de la salud.

Kenya: recomendada para trabajadores de campos de refugiados, y visitantes al área norte, frontera con Sudán y Uganda, zonas lejanas a las rutas habituales de turistas.

Angola, Burundi, Mozambique, Rwanda, Tanzania: aunque fuera del cinturón de meningitis, se han reportado casos en la época seca que en al caso de estos países es mayo-octubre (sur del Ecuador).

\section{Inmunizaciones de rutina}

\section{Tétanos/difteria/ pertussis}

Los adultos que no hayan recibido una dosis previa de vacuna pertussis acelular deben recibir por una vez la vacuna Tdap en vez del refuerzo con Td (En todos los países).

\section{Sarampión}

La vacuna está indicada en aquellos nacidos después de 1965, sin historia de la enfermedad o que no hayan recibido dos dosis de vacuna.

\section{Poliomielitis}

Está indicada la vacunación durante la infancia y una dosis de adulto (inactivada), excepto Malawi y Mozambique donde no es necesario el refuerzo.

\section{Malaria}

Esta área de África requiere de quimioprofilaxis para los viajeros, incluyendo visitas a zonas urbanas (excepto centro de Nairobi, Kenya.

Se requiere medidas de protección contra picadura de insectos en el atardecer y noche (repelentes, mosquiteros, insecticidas).

Los medicamentos sugeridos son: mefloquina, doxiciclina, proguanil/atavacuona.

\section{Cono sur de África}

Incluye: Namibia, Botswana, Zimbabwe, Sudáfrica, Lesotho, Madagascar, Mauricios.

\section{Posibles riesgos}

Prevenibles por vacunas: Hepatitis A y B, poliomielitis, rabia, fiebre tifoidea, influenza.
Otras enfermedades (no prevenibles por vacunas): Malaria, tripanosomiasis africana, dengue, esquistosomiasis, diarrea del viajero, tuberculosis, tifus exantemático (rickettsiasis).

\section{Fiebre amarilla}

En estos países no hay fiebre amarilla; sin embargo, existe el vector y sus autoridades exigen vacunación a viajeros provenientes de países endémicos de fiebre amarilla o que hayan pasado por uno de estos países.

\section{Fiebre tifoidea}

La vacunación está recomendada para Botswana, Lesotho, Madagascar, Namibia, Zimbabwe. En el caso de Sudáfrica y Mauricios sólo para aquellos viajeros con hábitos dietéticos aventureros, estadías prolongadas (especialmente en áreas rurales).

\section{Hepatitis $A$ y $B$}

Estadías extensas, trabajadores del área de la salud o en estadías cortas en aquellos viajeros que desean la máxima protección.

\section{Rabia}

Botswana: Hay riesgo en todo el país, sobre todo en el norte.

Lesotho, Madagascar, Namibia, Zimbabwe: Riesgo en todo el país, se recomienda vacunación pre-exposición para aquellas estadías prolongadas, en especial en niños.

Sudáfrica: Hay riesgo en todo el país, especialmente en las provincias de KwaZulu-Natal, Mpumalanga y provincias del este del cabo. Se recomienda para estadías prolongadas, especialmente en niños.

Mauricios: Es considerada libre de rabia por la OMS.

\section{Vacunaciones de rutina}

\section{Tétanos/difteria/ pertussis}

Los adultos que no hayan recibido una dosis previa de vacuna pertussis acelular deben recibir por una vez la vacuna Tdap en vez del refuerzo Td (En todos los países).

\section{Sarampión}

Vacunación indicada en aquellos nacidos después de 1965, sin historia de la enfermedad o que no hayan recibido dos dosis de vacuna.

\section{Poliomielitis}

Namibia: vacunación durante la infancia y una dosis de adulto (inactivada).

Botswana, Lesotho, Madagascar, Mauricios, Sudáfrica, Malawi, Mozambique Zimbabwe: no se requiere refuerzo en los adultos que viajan a estos países. 


\section{Malaria}

Botswana: Existe riesgo, fundamentalmente, de $P$. falciparum, en la zona norte del país, en especial el delta del río Okovango y la reserva de Chobe.

Madagascar, Zimbabwe: Existe riesgo, fundamentalmente, de $P$. falciparum, en todo el país incluyendo áreas urbanas, especialmente en zonas de la costa. En Zimbabwe el riesgo es mayor de noviembre a junio, excepto la zona de las cataratas Victoria donde el riesgo es todo el año. El riesgo es muy bajo en las ciudades de Harare y Bulawayo.

Namibia: el riesgo es todo el año en la zona norte en la vecindad de los ríos Kavango y Kunene y en las provincias fronterizas a Angola. El riesgo es de noviembre a junio en los parques de Etosha y Omaheke y la provincia de Otjozondjupa.

Sudáfrica: Sólo en la zona del noreste, donde está el sistema de parques Krüger además de las zonas bajas de las provincias Mpumalanga y KwaZulu-Natal. El resto del país carece de riesgo de malaria

Para detalles del riesgo de malaria consultar en http:// wwwnc.cdc.gov/travel/yellowbook/2010/chapter-2/ malaria-risk-information-and-prophylaxis.aspx 